\title{
Shocked water in the Cepheus E protostellar outflow
}

\author{
B. Lefloch ${ }^{1,2}$, J. Cernicharo ${ }^{2}$, S. Pacheco ${ }^{1}$, and C. Ceccarelli ${ }^{1}$ \\ ${ }^{1}$ UJF-Grenoble 1/CNRS-INSU, Institut de Planétologie et d'Astrophysique de Grenoble (IPAG) UMR 5274, Grenoble 38041, France \\ e-mail: lefloch@obs.ujf-grenoble.fr \\ ${ }^{2}$ Laboratorio de Astrofísica Molecular, Departamento de Astrofísica, Centro de Astrobiologia, INTA, Ctra de Torrejón a Ajalvir, \\ km 4, 28850 Torrejón de Ardoz, 28850 Madrid, Spain
}

Received 1 December 2010 / Accepted 10 January 2011

\section{ABSTRACT}

\begin{abstract}
Context. Previous far-infrared observations at low-angular resolution have reported the presence of water associated with low-velocity outflow shocks and protostellar envelopes.

Aims. We want to elucidate the origin of water emission in protostellar systems.

Methods. The outflow driven by the intermediate-mass class 0 protostar Cep E is among the most luminous outflows detected so far. Using the IRAM 30 m telescope, we searched for and detected the $\mathrm{p}-\mathrm{H}_{2} \mathrm{O} 3_{13}-2_{20}$ line emission at $183 \mathrm{GHz}$ in the Cep E star-forming core. The emission arises from high-velocity gas close to the protostar, which is unresolved in the main beam of the telescope. Complementary observations at $2^{\prime \prime}$ resolution with the Plateau de Bure interferometer helped establish the origin of the emission detected and the physical conditions in the emitting gas. The water line profile and its spatial distribution are very similar to those of $\mathrm{SiO}$. We find that the $\mathrm{H}_{2} \mathrm{O}$ emission arises from warm $(\sim 200 \mathrm{~K})$, dense $\left((1-2) \times 10^{6} \mathrm{~cm}^{-3}\right)$ gas, and its abundance is enhanced by one to two orders of magnitude with respect to the protostellar envelope.

Results. We detect water emission in strong shocks from the high-velocity jet at $1000 \mathrm{AU}$ from the protostar. Despite the large beam size of the telescope, such emission should be detectable with Herschel.
\end{abstract}

Key words. stars: formation - ISM: molecules - ISM: jets and outflows - ISM: individual objects: Cep E

\section{Introduction}

Water is a key molecule not only for oxygen chemistry in the interstellar gas, but also in the dynamical evolution of star-forming regions, because it is one of the main gas coolants (Kaufman $\&$ Neufeld 1996; Bergin et al. 1998). Whereas its abundance is very low in dark clouds, prestellar cores, and starless regions (from less than $10^{-8}$ to a few $10^{-7}$; Bergin \& Snell 2002; Moneti et al. 2001), it can be greatly enhanced in star-forming regions, through the sputtering of frozen water from grain mantles and through high-temperature, sensitive reactions in the gas phase (Elitzur \& de Jong 1978; Ceccarelli et al. 1996; Kaufmann \& Neufeld 1996). It has been proposed that such processes are driven by the heating of the nascent stars (hot core/corino region) or by shocks either in the accretion region in the inner protostellar core or in the outflow carrying away the angular momentum of the envelope. Previous observations of water lines obtained with the Infrared Space Observatory have substantially confirmed these theoretical expectations (see e.g. Giannini et al. 2001; Ceccarelli et al. 2000; van Dishoeck 2004, for a review). However, many details are still missing and the relative efficiency of these mechanisms is not known, so that the origin of the water emission in protostellar regions remains a puzzle to be solved. One obstacle is indeed the relatively low spatial resolution of these observations.

Most of the $\mathrm{H}_{2} \mathrm{O}$ lines cannot be observed from ground because of strong atmospheric absorption. The para- $\mathrm{H}_{2} \mathrm{O}$ line $3_{13}-2_{20}$ at $183.3 \mathrm{GHz}$ is an exception since it can be enhanced by the masing effect even in regions of moderate excitations. Using the IRAM 30 m telescope, Cernicharo et al. (1990, 1994) detected large-scale $\mathrm{p}-\mathrm{H}_{2} \mathrm{O}_{3}-2_{20}$ line emission in giant molecular clouds and star-forming regions and they showed that this line can be used to investigate the spatial distribution of $\mathrm{H}_{2} \mathrm{O}$ in protostellar environments at an angular resolution comparable to the size of the protostellar cores (14" at the IRAM $30 \mathrm{~m}$ telescope). Observing the low-mass protostellar outflows HH 7-11 and L1448, Cernicharo et al. (1996) detected water line emission arising from shocks associated with the Herbig-Haro objects in the outflows.

In this Letter, we report on observations of the $\mathrm{p}-\mathrm{H}_{2} \mathrm{O} 3_{13}-2_{20}$ line in the star-forming core CepE with the IRAM $30 \mathrm{~m}$ telescope ${ }^{1}$. Located at $730 \mathrm{pc}$, it is a relatively nearby intermediate-mass Class 0 source of $100 L_{\odot}$ and a mass of $18 M_{\odot}$, intensively studied at $\mathrm{mm}$ and IR wavelengths (Moro-Martin et al. 2001; Noriega-Crespo et al. 2004; Lefloch et al. 1996, hereafter L96). The protostar drives an exceptionally powerful outflow, whose southern lobe is terminated by the Herbig-Haro object HH 377. Far-infrared observations at low spectral and angular resolution with ISO have revealed large amounts of water, which Giannini et al. (2001) interpret as arising from low-velocity shocks, whose far-infrared luminosity amounts to $L \sim 3 L_{\odot}$.

Observations of the millimeter lines of $\mathrm{SiO}$ observations reveal the presence of strong shocks along the outflow (L96). Because it is usually undetected in the cold, quiescent molecular gas, $\mathrm{SiO}$ is a particularly good tracer of shocks that are strong enough to release refractory elements from grain cores. Recent

1 Based on observations carried out with the IRAM 30 m Telescope. IRAM is supported by INSU/CNRS (France), MPG (Germany), and IGN (Spain). 
Herschel observations suggest that $\mathrm{SiO}$ is also a good tracer of the water-emitting shock regions (Lefloch et al. 2010; Nisini et al. 2010). We present observations of the high-excitation $\mathrm{SiO}$ $J=8 \rightarrow 7$ line towards the protostar.

\section{Observations}

\subsection{Water observation}

We observed the line of para- $\mathrm{H}_{2} \mathrm{O} 3_{13} \rightarrow 2_{20}$ at $183.3 \mathrm{GHz}$ at the IRAM 30 m telescope in January 2009, near the transit of Cep E between $61^{\circ}$ and $65^{\circ}$ of elevation. Both receivers at $2 \mathrm{~mm}$ were used simultaneously. Observations were carried out in wobbler switching mode with a throw of $180^{\prime \prime}$. Filterbanks with $1 \mathrm{MHz}$ spectral resolution and an autocorrelator providing a spectral resolution of $1.25 \mathrm{MHz}\left(2 \mathrm{~km} \mathrm{~s}^{-1}\right)$ were used as spectrometers. Weather conditions were very good and stable, with an atmospheric optical depth $\approx 1.10$ along the line of sight. Calibration was carefully monitored every 4 minutes between two integration scans on Cep E. Water vapor and system temperatures were found to remain stable, in the range 1650-1800 K. Any fluctuation of the sky emissivity between the on and off positions would result in a poor spectroscopic baseline and detection of the mesospheric water line, which is not the case in the present observations. Pointing was checked against nearby quasars every hour and has an accuracy of $\approx 2^{\prime \prime}$. At the frequency of the $\mathrm{H}_{2} \mathrm{O}$ line, the main-beam efficiency of the telescope is 0.63 and the half-power beamwidth is $13.2^{\prime \prime}$. We did a small oversampled map at $6^{\prime \prime}$ sampling of the southern lobe of the outflow, centered on the nominal position of the protostar $23^{\mathrm{h}} 03^{\mathrm{m}} 13.0^{\mathrm{s}}$ $+61^{\circ} 42^{\prime} 21^{\prime \prime}$ (J2000). The typical $\mathrm{rms}$ is $100 \mathrm{mK}$ per velocity interval of $2 \mathrm{~km} \mathrm{~s}^{-1}$.

\subsection{SiO observations}

The $\mathrm{SiO} J=8 \rightarrow 7$ transition at $347.33006 \mathrm{GHz}$ was observed towards the protostar at the JCMT in August 2005. The weather conditions were good with an average system temperature $T_{\text {sys }} \sim$ $400 \mathrm{~K}$. Observations were carried out with a nutating secondary, adopting a throw of $60^{\prime \prime}$. The DBS was used as spectrometer, providing a spectral resolution of $625 \mathrm{kHz}$. The resolution was degraded to $3.75 \mathrm{MHz}$, corresponding to a final velocity resolution of about $3.2 \mathrm{~km} \mathrm{~s}^{-1}$. At the frequency of the $\mathrm{SiO}$ line, the main-beam efficiency of the telescope is about 0.60 and the halfpower beamwidth is $\simeq 14.2^{\prime \prime}$, very similar to the telescope parameters of the $\mathrm{p}-\mathrm{H}_{2} \mathrm{O}$ observations. Complementary interferometric observations at $2^{\prime \prime}$ resolution with the Plateau de Bure in the $\mathrm{SiO}$ $J=2 \rightarrow 1$ transition provide us with the spatial distribution of the emission and its excitation conditions. These observations will be presented and discussed in detail in a forthcoming paper (Lefloch et al., in prep.).

\section{Results and discussion}

\subsection{Water emission in CepE}

The map of the $\mathrm{p}-\mathrm{H}_{2} \mathrm{O}_{3}-2_{20}$ emission in Cep E is displayed in the left panel of Fig. 1. Emission is detected only towards the protostellar core, in a region unresolved by the main beam of the IRAM 30 m telescope. In particular, we do not detect any $\mathrm{H}_{2} \mathrm{O}$ emission towards $\mathrm{HH} 77$, the southern bowshock at offset position $\left(-5^{\prime \prime},-15^{\prime \prime}\right)$, associated with bright emission in the lines of $\mathrm{SiO}$ and $\mathrm{H}_{2}$ (L96). To improve the signal-to-noise ratio of the data, we averaged the emission of the water line over the inner
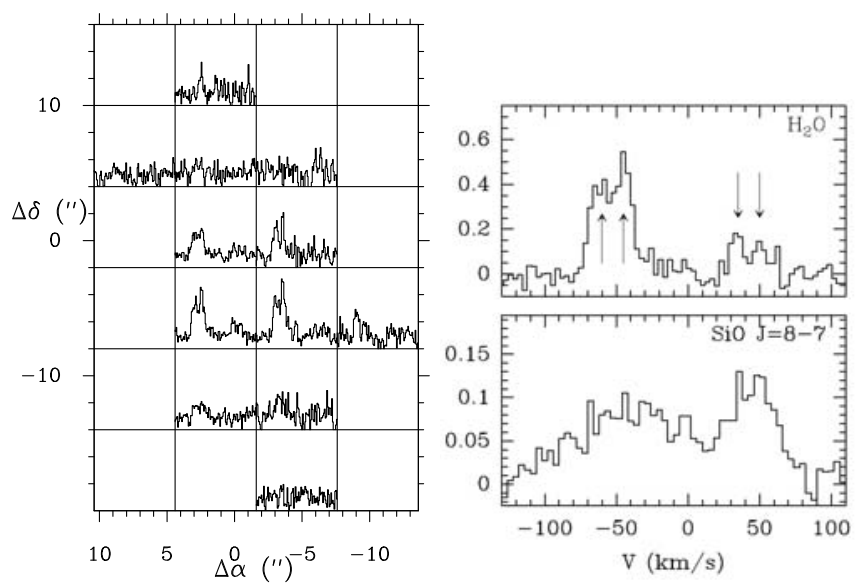

Fig. 1. (Left) Map of the $\mathrm{p}-\mathrm{H}_{2} \mathrm{O} 3_{13}-2_{20}$ emission between -110 and $+70 \mathrm{~km} \mathrm{~s}^{-1}$ in the outflow of Cep E obtained with the IRAM $30 \mathrm{~m}$ telescope. Coordinates are in arcsec offsets with respect to the nominal position of the protostar. (Right) $\mathrm{H}_{2} \mathrm{O}$ and $\mathrm{SiO} J=8 \rightarrow 7$ emission detected towards the protostellar source. The $\mathrm{H}_{2} \mathrm{O}$ emission is averaged over a region of $6^{\prime \prime} \times 6^{\prime \prime}$ around the protostar. Intensities are expressed in units of antenna temperature. The different components of the spectrum are marked with arrows.

core, in the range $\Delta \alpha=\left[1^{\prime \prime} ;-5^{\prime \prime}\right] \Delta \delta=\left[1^{\prime \prime} ;-5^{\prime \prime}\right]$. The averaged spectrum is displayed in the right panel of Fig. 1, and the final rms noise is $30 \mathrm{mK}\left(T_{\mathrm{A}}^{*}\right)$ per interval of $2 \mathrm{~km} \mathrm{~s}^{-1}$.

Two bright components $(\sim 1 \mathrm{~K})$ with broad linewidths $\left(\sim 10 \mathrm{~km} \mathrm{~s}^{-1}\right)$ are detected at about $v_{\mathrm{lsr}} \approx-65$ and $-45 \mathrm{~km} \mathrm{~s}^{-1}$ in the direction of the protostar. Two other, weaker, $\mathrm{H}_{2} \mathrm{O}$ components are detected at approximately symmetric (redshifted) velocities $v_{\mathrm{lsr}} \approx+34$ and $+50 \mathrm{~km} \mathrm{~s}^{-1}$. We do not detect any emission at the velocity of the protostellar envelope $\left(v_{\mathrm{lsr}}=\right.$ $-10.9 \mathrm{~km} \mathrm{~s}^{-1}$; L96), hence excluding any contribution from the hot corino to the water emission. The presence of both blue- and redshifted components in the water line spectrum indicates that the emission arises from the central protostellar region. This is because there is barely any overlap in the plane of the sky between the blue and redshifted wings of the Cep E outflow, except in the central protostellar region as a consequence of the limited angular resolution of the observations (see Figs. 1, 2; also L96).

The spectrum of the $\mathrm{SiO} J=8 \rightarrow 7$ emission in the direction of the protostar is displayed in Fig. 1 and looks very similar to the $\mathrm{p}-\mathrm{H}_{2} \mathrm{O} 3_{13}-2_{20}$ line spectrum. Resemblance between both emissions is even more striking when comparing the water spectrum with the $J=2 \rightarrow 1$ emission observed with the PdBI (Fig. 2). This suggests that the emission of both tracers arises from the same region. Observations of the $J=2 \rightarrow 1$ transition with the PdBI of the central protostellar regions (Lefloch et al., in prep.) show that the $\mathrm{SiO}$ emission arises from two compact clumps, in the blue- and redshifted gas, respectively, located at $\approx 1.5^{\prime \prime}$ from the protostar. They have a typical size of $2.5^{\prime \prime}$ $\left(2.3 \times 10^{16} \mathrm{~cm}\right)$, and a transverse size of $1^{\prime \prime}$ (Fig. 2). Their similar physical properties (size, velocity, distance) suggest they are related to an episodic ejection phenomenon. In what follows, we assume that both the $\mathrm{H}_{2} \mathrm{O}$ and the $\mathrm{SiO}$ emissions have the same size.

\subsection{Physical conditions}

The gas kinetic temperature $T_{\mathrm{k}}$ in the redshifted, high-velocity jet was previously determined by Hatchell et al. (1999) from JCMT observations of the CO $J=4 \rightarrow 3$ and $J=2 \rightarrow 1$ lines. 


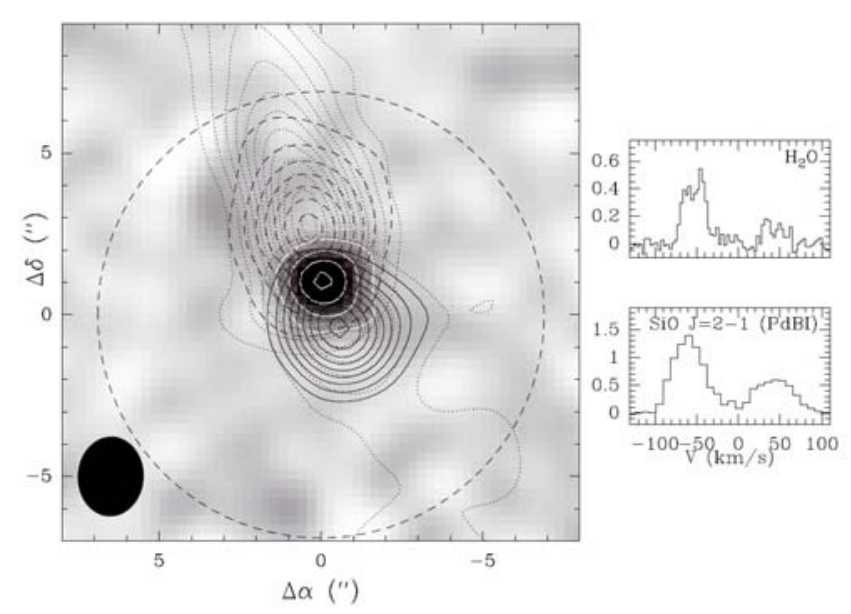

Fig. 2. (Left) Map of the velocity-integrated $\mathrm{SiO}$ emission in the Cep E jet obtained at $2^{\prime \prime}$ resolution with the Plateau de Bure interferometer (PdBI; Lefloch et al., in prep.). The $\mathrm{SiO} J=2 \rightarrow 1$ emission integrated between +40 and $+80 \mathrm{~km} \mathrm{~s}^{-1}\left(-90\right.$ and $\left.-40 \mathrm{~km} \mathrm{~s}^{-1}\right)$ is drawn in solid (dashed) contours, superposed on the $3 \mathrm{~mm}$ continuum emission map (grayscale and white contours). First contour and contour interval of the jet emission are $10^{-2}$ and $5 \times 10^{-3}\left(10^{-2}\right) \mathrm{Jy} /$ beam for the redshifted (blueshifted) emission. First contour and contour interval of the $3 \mathrm{~mm}$ continuum emission is $2 \times 10^{-3} \mathrm{Jy} /$ beam. The main beam of the IRAM $30 \mathrm{~m}$ telescope at the frequency of the $\mathrm{p}-\mathrm{H}_{2} \mathrm{O} 3_{13}-2_{20}$ line is marked by a circle (dashed). The synthesized beam of the PdBI $\left(2.4^{\prime \prime} \times 2.0^{\prime \prime}\right)$ is indicated as a filled ellipse in the bottom right corner. (Right) Comparison of the PdBI SiO $J=2 \rightarrow 1$ and the average $\mathrm{p}-\mathrm{H}_{2} \mathrm{O} 3_{13}-2_{20}$ spectra towards the protostar. Intensities are expressed in units of antenna temperature.

Unfortunately, their analysis is biased by a highly overestimated value of the main-beam efficiency $\eta_{\mathrm{MB}}$ of the $J=4 \rightarrow 3$ observations, poorly known at that time, leading to an underestimate of $T_{\mathrm{k}}(\approx 50 \mathrm{~K})$. Based on their observations and adopting a standard value consistent with the subsequent determinations from point-like calibrators ${ }^{2}, \eta_{\mathrm{MB}}=0.35$, we obtain the jet kinetic temperature and column density, $T_{\mathrm{k}} \simeq 120 \mathrm{~K}$ and $N(\mathrm{CO}) \simeq 2.5 \times 10^{17} \mathrm{~cm}^{-2}$, respectively (see also L96). Adopting a standard $\mathrm{CO}$ abundance in the gas, we then derive the sourceaveraged gas column-density $N\left(\mathrm{H}_{2}\right) \simeq 2.5 \times 10^{21} \mathrm{~cm}^{-2}$. We adopt these values of $T_{\mathrm{k}}$ and $\mathrm{N}(\mathrm{CO})$ for both water-emitting clumps at blue- and redshifted velocities.

The gas density and column density in the water-emitting clumps were derived from modeling the $\mathrm{H}_{2} \mathrm{O}$ and $\mathrm{SiO}$ line brightness temperatures in the large-velocity gradient (LVG) approach. We adopted the collisional coefficients of Faure et al. (2007) and Dayou \& Balança (2006), respectively. The gas density can be constrained reasonably well from the ratio of the $\mathrm{SiO} J=8 \rightarrow 7$ to $J=3 \rightarrow 2$ brightness temperatures. This ratio was computed using the $\mathrm{SiO} J=3 \rightarrow 2$ observations in the Cep E protostellar core obtained by L96 (see also Fig. 3). We note that the source is unresolved by the single-dish JCMT and IRAM 30 m observations; therefore, we approximated the ratio of the line brightness temperatures by the ratio of the mainbeam brightness temperatures corrected for the difference of main-beam solid angles $R(8-7 / 3-2)$. A description of the radiative transfer code and the procedure applied to deal with the masing effect is presented in Cernicharo et al. (1994) and González-Alfonso \& Cernicharo (1993).

\footnotetext{
2 http://www . jach.hawai i.edu/JCMT/spectral\$_\$line/ Standards/
}
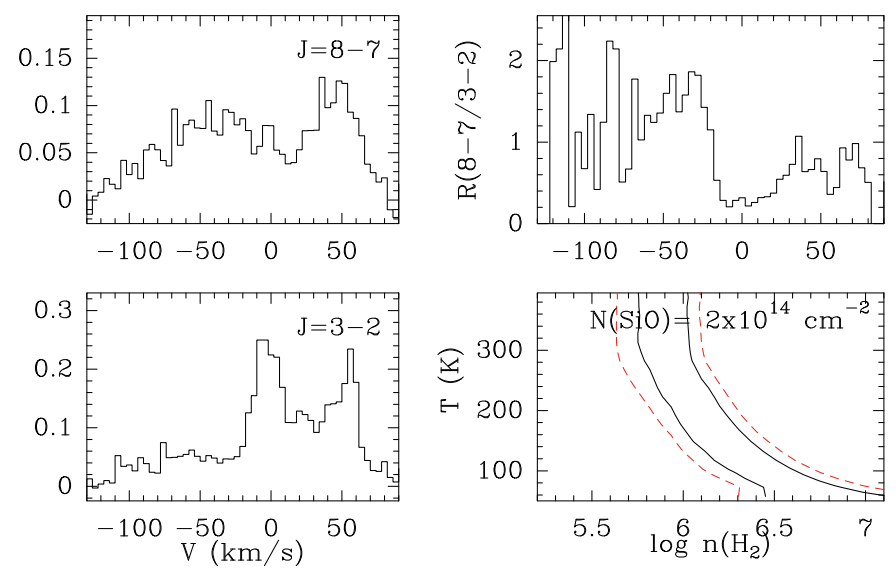

Fig. 3. (Left) $\mathrm{SiO}$ emission observed in the $J=3 \rightarrow 2$ (bottom) and $J=8 \rightarrow 7$ transitions towards the protostellar source. Fluxes are expressed in units of main-beam temperature. (Right) Observed variations of the ratio of the $\mathrm{CO} J=8 \rightarrow 7$ to $J=3 \rightarrow 2$ brigthness temperatures $R(8-7 / 3-2)$ as a function of velocity (top) and predicted variations as a function of the gas density $n\left(\mathrm{H}_{2}\right)$ and the kinetic temperature $T_{\mathrm{k}}$ (bottom) for a typical column density $N(\mathrm{SiO})=2 \times 10^{14} \mathrm{~cm}^{-2}$. The contour level $R=0.7,1.5$ are drawn with a solid black line; the contours $R=0.5,1.8$ are drawn with a dashed red line and account for an uncertainty of $30 \%$.

The top right panel of Fig. 3 displays the variations of $R(8-7 / 3-2)$. The ratio varies little in the low-velocity gas and is about 0.2. It increases as a function of velocity and reaches values up to 0.7 and 1.5 in the redshifted and blueshifted gas, respectively. The variations of $R(8-7 / 3-2)$ as a function of $T_{\mathrm{k}}$ and $n\left(\mathrm{H}_{2}\right)$ were computed for a typical column density $N(\mathrm{SiO})=$ $2 \times 10^{14} \mathrm{~cm}^{-2}$ and a linewidth $\Delta V=10 \mathrm{~km} \mathrm{~s}^{-1}$, and they are displayed in Fig. 3. With the constraint $T_{\mathrm{k}} \geq 100 \mathrm{~K}$, it comes out that $n\left(\mathrm{H}_{2}\right)$ must be about $1.0 \times 10^{6} \mathrm{~cm}^{-3}$ in order to account for the line intensity ratio in the redshifted gas. The density in the blueshifted gas is slightly higher $\approx 2 \times 10^{6} \mathrm{~cm}^{-3}$. These calculations show that the ratio depends weakly on $T_{\mathrm{k}}$ above $100 \mathrm{~K}$. For these values of $n\left(\mathrm{H}_{2}\right)$ and the kinetic temperature estimated above, we estimate the source-averaged column density necessary to account for the $J=8 \rightarrow 7$ line intensity $N(\mathrm{SiO})=(2.0-3.3) \times 10^{14} \mathrm{~cm}^{-2}$ for gas at $120 \mathrm{~K}$ in the blue- and redshifted components. Under such physical conditions both $\mathrm{SiO}$ transitions are optically thin, so that $R(8-7 / 3-2)$ depends weakly on the adopted value of the gas column density. From comparison with $N(\mathrm{CO})$ and assuming a standard abundance $[\mathrm{CO}] /\left[\mathrm{H}_{2}\right]=10^{-4}$, we obtain the relative $\mathrm{SiO}$ abundance in the shock: $[\mathrm{SiO}] /\left[\mathrm{H}_{2}\right] \simeq 1.0 \times 10^{-7}$, similar to the abundances measured in other Class 0 protostellar outflows (Lefloch et al. 1998).

The para-water (source-averaged) column density $N\left(\mathrm{p}-\mathrm{H}_{2} \mathrm{O}\right)$ was determined from modeling the line brightness temperature $T_{\mathrm{B}}$. The latter was derived from the main-beam brightness temperature $T_{\mathrm{MB}}=T_{\mathrm{B}} \times f f$, where the filling factor $f f$ was obtained assuming that the $\mathrm{H}_{2} \mathrm{O}$ and $\mathrm{SiO} J=2 \rightarrow 1$ emitting regions have similar sizes, in agreement with Sect. 3.1. We find $T_{\mathrm{B}}=60-80 \mathrm{~K}$ and $15-29 \mathrm{~K}$ in the blueshifted and redshifted gas, respectively. The brightness temperature of the $\mathrm{p}-\mathrm{H}_{2} \mathrm{O}_{3}{ }_{13}-2_{20}$ line was computed for a wide range of physical conditions, adopting a typical linewidth $\Delta v=10 \mathrm{~km} \mathrm{~s}^{-1}$ : $T_{\mathrm{k}}$ between 50 and $200 \mathrm{~K}, n\left(\mathrm{H}_{2}\right)$ in the range $10^{3}-10^{8} \mathrm{~cm}^{-3}$ and $N\left(\mathrm{p}-\mathrm{H}_{2} \mathrm{O}\right)$ in the range $3 \times 10^{16}$ to $10^{19} \mathrm{~cm}^{-2}$. The results of the calculations are presented in Fig. 4. 


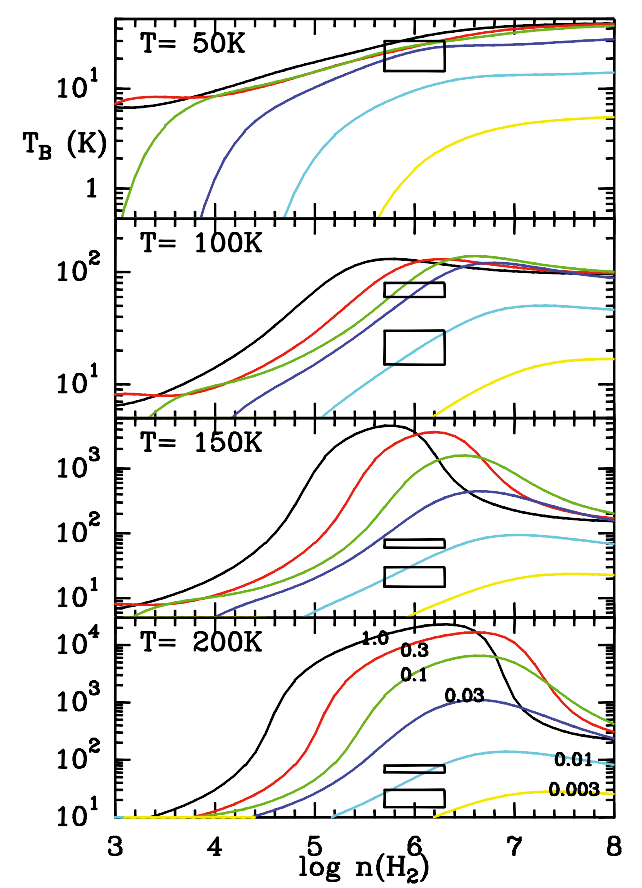

Fig. 4. Predictions of the $\mathrm{p}-\mathrm{H}_{2} \mathrm{O} 3_{13}-2_{20}$ line intensity, in units of brightness temperature, for gas with density $n\left(\mathrm{H}_{2}\right)$ in the range $10^{3}-10^{8} \mathrm{~cm}^{-3}$, temperature in the range 50-200 K, for column densities of para-water equal to 0.003 (bottom curve), $0.01,0.03,0.1,0.3,1.0 \times 10^{19} \mathrm{~cm}^{-2}$ (top curve). The range of $T_{\mathrm{B}}-n\left(\mathrm{H}_{2}\right)$ determined in the water-emitting clumps is delineated by rectangles.

It comes out that the kinetic temperature $T_{\mathrm{k}}$ has to be higher than $100 \mathrm{~K}$ to account for brightness temperatures of $60-80 \mathrm{~K}$. Our calculations indicate a $\mathrm{p}-\mathrm{H}_{2} \mathrm{O}$ column density in the range $(0.3-2.0) \times 10^{17} \mathrm{~cm}^{-2}$ for $T_{\mathrm{k}} \simeq 200 \mathrm{~K}$. Assuming an ortho-topara ratio of 3 , we derive $N\left(\mathrm{H}_{2} \mathrm{O}\right)=(1.2-8.0) \times 10^{17} \mathrm{~cm}^{-2}$, and using the $\mathrm{H}_{2}$ column density derived in the high-velocity clumps, we obtain the water abundance $X\left(\mathrm{H}_{2} \mathrm{O}\right)=(0.5-3.2) \times 10^{-4}$. In this range of physical conditions, the line optical depth $\tau$ is small $\approx-1$ and shows evidence for the weak masing amplification of $\mathrm{p}-\mathrm{H}_{2} \mathrm{O} 3_{13}-2_{20}$ line. Suprathermal excitation of the line would require much higher $\mathrm{H}_{2} \mathrm{O}$ column densities. This is possible if the emission arises from a small region of subarsec size (see e.g. Cernicharo et al. 1994) and is very sharp. The line $T_{\mathrm{B}}$ would then look weaker as a result of the dilution in the $2 \mathrm{~km} \mathrm{~s}^{-1}$ channels of the spectrometer.

Moro-Martin et al. (2001) analyzed the far-infrared emission lines of $\mathrm{CO}$ and $\mathrm{H}_{2} \mathrm{O}$ in Cep $\mathrm{E}$, as observed with ISO. Like Giannini et al. (2001), they found that the line emission can be accounted for by an extended component of low-density gas $\left(2 \times 10^{4} \mathrm{~cm}^{-3}\right)$ at $\sim 1000 \mathrm{~K}$. They show that the $\mathrm{CO}$ and $\mathrm{H}_{2} \mathrm{O}$ lines could be reproduced equally well by "warm" gas at a much lower temperature $(\sim 200 \mathrm{~K})$ and higher density $\left(2 \times 10^{6} \mathrm{~cm}^{-3}\right)$. The physical conditions we derive for the $\mathrm{p}-\mathrm{H}_{2} \mathrm{O} 3_{13}-2_{20}$ emitting region are actually in rather good agreement with this "warm" solution and inconsistent with the low-density "hot" solution.

\subsection{Shocked water in CepE}

Crimier et al. (2010) determined the physical conditions in the protostellar envelope of CepE envelope and found $n\left(\mathrm{H}_{2}\right)=$ $1.0 \times 10^{7} \mathrm{~cm}^{-3}$ and $T_{\mathrm{k}} \sim 50 \mathrm{~K}$, respectively, at the location of the water-emitting clumps, i.e. $1000 \mathrm{AU}\left(1.4^{\prime \prime}\right)$ from the protostar. No $\mathrm{p}-\mathrm{H}_{2} \mathrm{O} 3_{13}-2_{20}$ emission is detected from the envelopeat the $2 \sigma$ level $=3 \mathrm{~K}\left(T_{\mathrm{B}}\right)$. From Fig. 4 , we conclude that $N\left(\mathrm{H}_{2} \mathrm{O}\right)<10^{16} \mathrm{~cm}^{-2}$ in the inner protostellar envelope, and the water abundance is enhanced by 1 to 2 orders of magnitude in the high-velocity clumps. The authors estimate a radius of $\approx 220 \mathrm{AU}\left(0.3^{\prime \prime}\right)$ for the hot corino region, and the density is predicted very high $\sim 10^{8} \mathrm{~cm}^{-3}$; again, no masing effect is to be expected, in agreement with our observations.

The water-emitting clumps are located along the highvelocity jet, which suggests that they are closely related with the jet (Fig. 2). One possibility is that these clumps are tracing the jet/envelope interaction. However, the density of the protostellar envelope, as estimated by Crimier et al. (2010) at the location of the water emitting-clumps $\left(n\left(\mathrm{H}_{2}\right)=1.0 \times 10^{7} \mathrm{~cm}^{-3}\right)$, is about one order of magnitude higher than the density measured in the shocked gas $\left(10^{6} \mathrm{~cm}^{-3}\right)$, in apparent contradiction with this hypothesis. The structure of the envelope could be actually more complex than the simple power-law distribution derived by Crimier et al. (2010), as proposed that multiple protostars are embedded inside the core (Ladd \& Hodapp 1997). Direct observational evidence for several condensations is still missing, however. Alternatively, we speculate that the water emission could arise from internal shocks in the entrained material of the flow. Interferometric observations at subarcsec scale in the inner core $(r<1000 \mathrm{AU})$ are needed to determine the gas density and velocity field in order to determine the location and the origin of the water-emitting shocks, as well as their relation with the jet.

Simple calculations in the LVG approximation predict that the $\mathrm{H}_{2} \mathrm{O}$ emission from the high-velocity clumps should be easily detected in several ortho- and para- $\mathrm{H}_{2} \mathrm{O}$ transitions with the high-resolution spectrometer HIFI onboard Herschel. Such observations will allow us to better constrain the shock physical conditions (see e.g. Flower \& Pineau des Forets 2010).

Acknowledgements. We thank an anonymous referee and the Editor for comments that helped to improve the manuscript a lot. This project was supported by the research grant SAB2009-0011 of the Spanish Ministry of Education.

\section{References}

Bergin, E. A., \& Snell, R. L. 2002, ApJ, 581, L105

Bergin, E. A., Neufeld, D. A., \& Melnick, G. J. 1998, ApJ, 499, 777

Ceccarelli, C., Hollenbach, D., \& Tielenas, A. G. G. M. 1996, ApJ, 471, 400

Ceccarelli, C., Castets, A., Caux, E., et al. 2000, ApJ, 355, 1129

Cernicharo, J., Thum, C., Hein, H., et al., A\&A, 1990, 231, L15

Cernicharo, J., Gonzalez-Alfonso, E., \& Alcolea, J. 1994, 432, L59

Cernicharo, J., Bachiller, R., \& Gonzalez-Alfonso, E. 1996, A\&A, 305, L5

Crimier, N., Ceccarelli, C., \& Alonso-Albi, T., et al. 2010, A\&A, 516, A102

Dayou, F., \& Balança, C. 2006, A\&A, 459, 297

Eisloeffel, J., Smith, M. D., \& Davis, C.J. 1996, AJ, 112, 2086

Elitzur, M., \& de Jong, T., 1978, A\&A, 67, 323

Faure, A., Crimier, N., Ceccarelli, C., et al. 2007, A\&A, 472, 1029

Flower, D. R., \& Pineau des Forets, G. 2010, MNRAS, 406, 1745

Giannini, T., Nisini, B., \& Lorenzetti, D. 2001, ApJ, 555, 40

Kaufman, M., \& Neufeld, D. 1996, ApJ, 456, 611

Ladd, E. F., \& Hodapp, K.-W. 1997, ApJ, 475, 749

Lefloch, B., Eisloeffel, J., \& Lazareff, B. 1996, A\&A, 313, L17 (L96)

Lefloch, B., Castets A., Cernicharo, J., et al. 1998, A\&A, 504, L109

Moneti, A., Cernicharo J., \& Pardo J. R. 2001, ApJ, 549, L203

Moro-Martín, A., Noriega-Crespo, A., Molinari, S., et al., 2001, ApJ, 555, 146

Nisini, B., Benedettini, M., Codella, C. et al. 2010, A\&A, 518, L120

Noriega-Crespo, A., Moro-Martin, A., \& Carey, S. 2004, ApJS, 154, 402

Turner, B. E., Chan, Kin-Wing, Green, S., et al. 1992, ApJ, 399, 114

van Dishoeck, E. F. 2004, ARA\&A, 42, 119 